\section{Medicamentos prescritos aos idosos em uma capital do Sul do Brasil e a Relação Municipal de Medicamentos}

\author{
Medicines prescribed to the elderly in a city in \\ southern Brazil and the Municipal Medicines List
}

\author{
Marina Meneses Aziz 1 \\ Maria Cristina Marino Calvo 1 \\ Eleonora d'Orsi 1
}

\section{Introdução}

1 Universidade Federal

de Santa Catarina,

Florianópolis, Brasil.

Correspondência

M. M. Aziz

Universidade Federal de Santa

Catarina.

Rua Prof. José Brasilício

de Souza 100, apto. 703,

Florianópolis, SC 88036-530,

Brasil.

marinamaziz@gmail.com

\begin{abstract}
The aim of this study was to describe the medicines prescribed to elderly residents of Florianópolis, Santa Catarina State, Brazil, in the 30 days prior to the interview, and to compare them with the Municipal Medicines List (REMUME). A cross-sectional population-based household survey was conducted, in which 1,705 elderly respondents reported having used a total of 5,458 prescription drugs meeting the study criteria and corresponding to 374 different active ingredients. The most frequently used medicines were for the cardiovascular system, among which the most prevalent pharmacological class was lipidlowering drugs (although there were no examples of this drug class in the REMUME list). Despite some gaps, the majority of the drugs prescribed in Florianópolis coincided with the REMUME list. Prescriptions filled through the Unified National Health System were in greater conformity with the REMUME list.
\end{abstract}

Drug Utilization; Aged; Health Services Accessibility
O envelhecimento populacional no Brasil requer a adequação do sistema de saúde às novas necessidades apresentadas pelos idosos. Estudos farmacoepidemiológicos de base populacional têm mostrado que, com o avanço da idade, aumenta também o número de doenças crônicas, gerando a necessidade de utilização de uma quantidade maior de medicamentos 1 .

O comércio internacional de medicamentos cresceu rapidamente nos últimos anos, passando de US $\$ 5$ bilhões para US $\$ 120$ bilhões no período entre 1980 e 1999 2. Calculam-se os valores de vendas de US\$ 386 bilhões em 2001, US\$ 500 bilhões em 2004 3, US\$ 856 bilhões em 2010 e, conforme informações da IMS Health, este valor deve chegar a US $\$ 1,1$ trilhão até 2015 4 Com o aumento da produção, pesquisa e desenvolvimento de novos produtos, existe disponível no mercado uma grande quantidade de medicamentos comparáveis com relação à indicação, composição, mecanismo de ação, forma farmacêutica, apresentação, efeitos indesejáveis, eficácia e efetividade, porém, diferentes em maior ou menor grau ${ }^{5}$. Muitos desses produtos e suas indicações não atendem necessariamente ao conceito de medicamento essencial ou ao seu uso racional. Além disso, questões operacionais e financeiras impossibilitam aos sistemas de saúde prestar acesso a todos os medicamentos existentes. 
Uma importante estratégia para solucionar esse problema é a realização de uma avaliação entre esses medicamentos comparáveis, com base em forte evidência científica, elencandose uma lista de medicamentos na qual estariam os representantes dessa grande quantidade existente, obtendo-se daí uma lista de medicamentos essenciais. A formulação de listas também objetiva promover disponibilidade, acesso, sustentabilidade, qualidade e uso racional de medicamentos 6 .

No Brasil, a relação dos medicamentos considerados essenciais é a Relação Nacional de Medicamentos (RENAME), que se baseia "nas prioridades nacionais de saúde, bem como na segurança, na eficácia terapêutica comprovada, na qualidade e na disponibilidade dos produtos" 7 (p. 7). A finalidade da RENAME é servir "de instrumento básico para a elaboração das listas estaduais e municipais" - as Relações Estaduais de Medicamentos (RESME) e Relações Municipais de Medicamentos (REMUME) - além de orientar a prescrição médica, o direcionamento da produção farmacêutica e o desenvolvimento científico e tecnológico 7 .

Estudos com o objetivo de avaliar a adequação das listas de medicamentos fornecidos pelo SUS são importantes para identificação de problemas de acesso, e contribuem para a reorientação das políticas de medicamentos. A prescrição de medicamentos fora das listas pode refletir uma cultura de prescrição influenciada pela indústria farmacêutica, falta de conhecimento em relação aos medicamentos disponibilizados pelo SUS, mas também pode mostrar uma deficiência na formulação das listas de medicamentos fornecidos 8 .

O objetivo deste estudo foi descrever as características dos medicamentos prescritos aos idosos residentes na zona urbana do Município de Florianópolis, Santa Catarina, Brasil, e com base nessas informações, fazer uma comparação entre a REMUME e o que foi prescrito.

\section{Métodos}

Foi realizado um estudo transversal populacional de base domiciliar com idosos do Município de Florianópolis. O trabalho faz parte de um inquérito abrangente sobre as condições de saúde das pessoas idosas do Município de Florianópolis, realizado em 2009/2010, denominado EpiFloripa Idoso. A pesquisa foi financiada pelo Conselho Nacional de Desenvolvimento Cientifico e Tecnológico (CNPq) e aprovada pelo Comitê de Ética em Pesquisa da Universidade Federal de Santa Catarina (UFSC).
O Município de Florianópolis é a capital do Estado de Santa Catarina, com uma população estimada para o ano de 20099 de 408.163 habitantes, sendo $10,8 \%$ de idosos. A atenção ao idoso na rede municipal de saúde está organizada segundo diretrizes da Secretaria Municipal de Saúde 10, incluindo atendimento preferencial por profissionais de saúde na rede de atenção básica, atendimento e discussão de casos por profissional especializado em Geriatria, além de ações de promoção da saúde. A REMUME de Florianópolis incorpora todos os medicamentos da RENAME para distribuição na atenção básica, sendo que os medicamentos para média e alta complexidades são fornecidos pela Secretaria de Estado da Saúde. O acesso aos medicamentos previstos na REMUME ocorre para as prescrições do SUS e do sistema privado.

O tamanho da amostra do EpiFloripa Idoso foi calculado para estimar a prevalência com base em uma amostra casual simples, multiplicado por 2 (conglomerado em 2 estágios), e acréscimos de $20 \%$ para perdas previstas e $15 \%$ para controle de fatores de confusão. Para tal, foi usado o programa Epi Info, versão 6.04 (Centers for Disease Control and Prevention, Atlanta, Estados Unidos), com os seguintes parâmetros: tamanho da população igual a 44.460 , prevalência para o desfecho desconhecida (50\%), nível de 95\% de confiança, erro amostral igual a 4 pontos percentuais, sendo necessária uma amostra de, no mínimo, 1.599 pessoas.

O processo de seleção da amostra foi realizado por conglomerados em dois estágios. No primeiro, todos os 420 setores censitários urbanos da cidade foram colocados em ordem crescente de renda média mensal do chefe da família, sorteando-se sistematicamente 80 destes setores (oito setores em cada decil de renda).

As unidades do segundo estágio foram os domicílios. Uma etapa de atualização do número de domicílios em cada setor (arrolamento) fez-se necessária, uma vez que o censo mais recente havia sido realizado em 2000. Para tanto, os supervisores do estudo percorreram cada um dos setores censitários sorteados e procederam à contagem de todos os domicílios habitados, obedecendo às normas do Instituto Brasileiro de Geografia e Estatística (IBGE) 11. O número de domicílios nos setores variou de 61 a 725 .

Setores com menos de 150 domicílios foram agrupados e setores com mais de 500 domicílios foram divididos, respeitando o decil de renda correspondente, com a finalidade de diminuir o coeficiente de variação do número de domicílios por setor. Tal procedimento resultou em 83 setores censitários e reduziu o coeficiente de variação inicial de $52,7 \%$ para $35,2 \%$. Em seguida, foram 
sorteados sistematicamente cerca de 60 domicílios por setor censitário. Considerou-se elegíveis para serem entrevistados todos os idosos residentes nos domicílios sorteados.

Consideraram-se perdas os idosos que se encontravam impossibilitados de responder por motivo de viagem, e os que não foram localizados após quatro visitas, sendo pelo menos uma no período noturno e uma no final de semana; e recusas, os casos de sujeitos que se negaram a responder ao questionário por opção pessoal. Idosos com problemas cognitivos tiveram o questionário respondido por cuidadores/responsáveis $(\mathrm{n}=49)$.

A coleta de dados foi realizada por meio de um questionário padronizado e pré-testado, aplicado na forma de entrevistas face a face, utilizando-se o Personal Digital Assistants (PDA). Todos os entrevistados assinaram um Termo de Consentimento Livre e Esclarecido concordando em participar da pesquisa.

O trabalho de campo foi realizado entre setembro de 2009 e junho de 2010. As entrevistas foram realizadas por entrevistadoras com nível médio completo de escolaridade ou mais, devidamente treinadas sobre os procedimentos da pesquisa. O estudo-piloto incluiu 99 idosos residentes em setores não amostrados para a pesquisa. O trabalho de campo foi supervisionado por estudantes de cursos de pós-graduação.

Houve verificação semanal da consistência dos dados e controle de qualidade por meio de aplicação por telefone de um questionário reduzido, em $10 \%$ das entrevistas selecionadas aleatoriamente.

A coleta de dados foi realizada pelo nome comercial dos medicamentos, conforme embalagem ou receita médica. Para organização e classificação dos mesmos, verificou-se a substância ativa de cada medicamento na página da Internet http://www.consultaremedios.com.br. Para a padronização dos medicamentos conforme substância ativa e indicação de uso, os medicamentos também foram classificados conforme critérios estabelecidos pelo sistema de classificação ATC (Anatomical Therapeutic Chemical), criado pela WHO Collaborating Centre for Drug Statistics Methodology. Não foram consideradas as formas de absorção (liberação controlada etc.), concentração e forma farmacêutica.

Também perguntou-se quem indicou o medicamento - médico ou dentista do SUS; médico ou dentista particular/plano de saúde; outros profissionais de saúde - além de outras opções que sugeriam automedicação.

A forma de obtenção do medicamento foi classificada em duas categorias: farmácia do posto/Policlínica/Hospital SUS, consideradas como obtidas pelo SUS; e outras, consideradas como compra (amostra grátis, Farmácia Popular, outras farmácias).

Foram incluídos nas análises todos os medicamentos industrializados, assim como as fórmulas magistrais. Foram excluídos aqueles não prescritos por médico/dentista, assim como aqueles com mais de 2 associações, fitoterápicos, homeopáticos, vitaminas/suplementos, hormônios femininos (pela variabilidade de fórmulas existentes no mercado, o que impossibilita uma comparação direta entre os medicamentos), lubrificantes oculares, curativos, produtos de higiene, cosméticos, alimentos (fibras e probióticos) e nomes incompreensíveis/inexistentes.

Os medicamentos foram classificados em constantes ou não na REMUME. No caso dos que não constavam na REMUME, foi verificado se existia alguma alternativa terapêutica a este medicamento, da mesma classe farmacológica ou grupo químico, pelo código ATC do medicamento.

A REMUME do Município de Florianópolis referente ao período da realização das entrevistas (setembro de 2009 a junho de 2010) foi fornecida pela Gerência de Assistência Farmacêutica da Secretaria Municipal de Saúde do Município de Florianópolis.

Os dados são apresentados na forma de tabelas, em frequência porcentual simples, seguida de análises descritiva e comparativa com estudos realizados em outras cidades e regiões. A análise foi realizada no programa SPSS 15.0 (SPSS Inc., Chicago, Estados Unidos) e no pacote estatístico Stata versão 9.0 (Stata Corp., College Station, Estados Unidos).

\section{Resultados}

Nos domicílios sorteados foram encontrados 1.911 idosos elegíveis, sendo efetivamente entrevistados 1.705 , resultando em uma taxa de resposta de $89,2 \%$ ( $n=206$ perdas/recusas). O controle de qualidade verificou que a reprodutibilidade de algumas questões utilizadas no estudo foi considerada satisfatória, com valores de kappa entre 0,6 e 0,9 para as variáveis selecionadas (vacina antigripal, diabetes, número de dentes, tabagismo, plano de saúde e cor da pele autorreferida).

Os idosos entrevistados relataram ter utilizado o total de 6.533 medicamentos nos 30 dias anteriores à entrevista. Foram desconsiderados $904(13,8 \%)$ medicamentos da análise por não se enquadrarem nos critérios de inclusão do estudo, e 171 (2,6\%) por não serem medicamentos indicados por médico/dentista, resultando em 
uma amostra de 5.458 medicamentos. Esses, correspondiam a 374 princípios ativos diferentes.

A Tabela 1 demonstra os grupos de medicamentos mais utilizados pelos idosos nos 30 dias anteriores à entrevista, de acordo com a classi- ficação anatômica (nível 1) e por grupo farmacológico (nível 3) da ATC, e presença ou não na REMUME. De todos os medicamentos prescritos, inclusive pelo sistema privado, apenas $39,6 \%$ não estão disponíveis na REMUME. Os medica-

Descrição dos medicamentos prescritos, utilizados nos 30 dias anteriores à entrevista, por ordem decrescente do número de utilizações, conforme classificação anatômica (nível 1) e grupo farmacológico (nível 3) da Anatomical Therapeutical Chemical Index (ATC), e presença (sim) ou ausência (não) na Relação Municipal de Medicamentos (REMUME). Projeto EpiFloripa Idoso, Florianópolis, Santa Catarina, Brasil, 2010.

\begin{tabular}{|c|c|c|c|c|c|c|}
\hline \multirow[t]{2}{*}{ Medicamento } & \multicolumn{2}{|c|}{ Sim } & \multicolumn{2}{|c|}{ Não } & \multicolumn{2}{|c|}{ Total } \\
\hline & $\mathbf{n}$ & $\%$ & $\mathbf{n}$ & $\%$ & $\mathbf{n}$ & $\%$ \\
\hline C - Sistema Cardiovascular & 1.549 & 60,6 & 1.006 & 39,4 & 2.555 & 46,8 \\
\hline C10A - Redutores do colesterol e triglicerídeos & - & - & 432 & 100,0 & 432 & 7,9 \\
\hline C09A - Inibidor da enzima conversora da angiotensina & 362 & 91,0 & 36 & 9,0 & 398 & 7,3 \\
\hline C07A - Betabloqueadores & 237 & 76,7 & 72 & 23,3 & 309 & 5,7 \\
\hline C03A - Diuréticos tiazídicos & 244 & 89,4 & 29 & 10,6 & 273 & 5,0 \\
\hline C09C - Antagonistas da angiotensina II & 183 & 74,4 & 63 & 25,6 & 246 & 4,5 \\
\hline C08C - Bloqueadores seletivos dos canais de cálcio com efeitos vasculares & 124 & 77,0 & 37 & 23,0 & 161 & 2,9 \\
\hline C01D - Vasodilatadores utilizados em doenças cardíacas & 66 & 57,9 & 48 & 42,1 & 114 & 2,1 \\
\hline C03C - Diuréticos de alça & 95 & 97,9 & 2 & 2,1 & 97 & 1,8 \\
\hline C09D - Antagonistas da angiotensina II em associações & 43 & 44,8 & 53 & 55,2 & 96 & 1,8 \\
\hline C03D - Agentes poupadores de potássio & 54 & 100,0 & - & - & 54 & 1,0 \\
\hline C08D - Bloqueadores seletivos dos canais de cálcio com efeitos cardíacos & 19 & 35,2 & 35 & 64,8 & 54 & 1,0 \\
\hline Outros & 122 & 38,0 & 199 & 62,0 & 321 & 5,9 \\
\hline A - Trato Alimentar e Metabolismo & 650 & 79,7 & 166 & 20,3 & 816 & 15,0 \\
\hline A10B - Fármacos hipoglicemiantes orais & 319 & 82,0 & 70 & 18,0 & 389 & 7,1 \\
\hline A02B - Medicamentos para tratamento de úlcera péptica e refluxo gastroesofageal & 272 & 87,5 & 39 & 12,5 & 311 & 5,7 \\
\hline Outros & 59 & 50,9 & 57 & 49,1 & 116 & 2,1 \\
\hline $\mathrm{N}$ - Sistema Nervoso & 330 & 42,0 & 456 & 58,0 & 786 & 14,4 \\
\hline N06A - Antidepressivos & 98 & 43,8 & 126 & 56,3 & 224 & 4,1 \\
\hline N02B - Outros analgésicos e antipiréticos & 144 & 92,9 & 11 & 7,1 & 155 & 2,8 \\
\hline N03A - Antiepiléticos & 33 & 28,2 & 84 & 71,8 & 117 & 2,1 \\
\hline N05B - Ansiolíticos & 22 & 21,6 & 80 & 78,4 & 102 & 1,9 \\
\hline N07C - Preparações antivertiginosas & 15 & 28,3 & 38 & 71,7 & 53 & 1,0 \\
\hline Outros & 18 & 13,3 & 117 & 86,7 & 135 & 2,5 \\
\hline B - Sangue e Órgãos Formadores de Sangue & 312 & 73,8 & 111 & 26,2 & 423 & 7,8 \\
\hline B01A - Antitrombótico & 312 & 73,9 & 110 & 26,1 & 422 & 7,7 \\
\hline Outros & - & - & 1 & 100,0 & 1 & 0,0 \\
\hline M - Sistema Musculoesquelético & 92 & 33,3 & 184 & 66,7 & 276 & 5,1 \\
\hline M01A - Anti-inflamatórios e antirreumáticos não esteroidais & 92 & 55,4 & 74 & 44,6 & 166 & 3,0 \\
\hline M05B - Medicamentos que afetam a estrutura óssea e mineralização & - & - & 60 & 100,0 & 60 & 1,1 \\
\hline Outros & - & - & 50 & 100,0 & 50 & 0,9 \\
\hline H - Preparados Hormonais Sistêmicos, exceto Hormônios Sexuais & 247 & 96,9 & 8 & 3,1 & 255 & 4,7 \\
\hline H03A - Hormônios tireoidianos & 227 & 100,0 & - & - & 227 & 4,2 \\
\hline Outros & 20 & 71,4 & 8 & 28,6 & 28 & 0,5 \\
\hline R - Aparelho Respiratório & 66 & 39,8 & 100 & 60,2 & 166 & 3,0 \\
\hline R03A - Adrenérgicos para inalação & 26 & 31,7 & 56 & 68,3 & 82 & 1,5 \\
\hline Outros & 40 & 47,6 & 44 & 52,4 & 84 & 1,5 \\
\hline Outros (J, D, G, L, P, S) & 51 & 28,2 & 130 & 71,8 & 181 & 3,3 \\
\hline Total & 3.297 & 60,4 & 2.161 & 39,6 & 5.458 & 100,0 \\
\hline
\end{tabular}


mentos indicados para o sistema cardiovascular foram os mais utilizados $(46,8 \%)$, seguidos, em ordem decrescente, pelos medicamentos do trato alimentar e metabolismo (15\%), com ação no sistema nervoso $(14,4 \%)$ e com ação no sangue e órgãos formadores de sangue $(7,8 \%)$.

Considerando o grupo farmacológico, entre os medicamentos mais consumidos estão os redutores de colesterol e triglicerídeos (7,9\%), antitrombóticos (7,7\%), inibidores da enzima conversora de angiotensina $(7,3 \%)$ e fármacos hipoglicemiantes orais $(7,1 \%)$.

Verifica-se que nenhum dos medicamentos redutores de colesterol e triglicerídeos, tampouco os medicamentos que atuam na estrutura óssea e mineralização, estão elencados na REMUME, apesar de o primeiro ser o grupo farmacológico mais utilizado pelos idosos do município.

Cerca de $60 \%$ dos medicamentos prescritos, tanto por profissionais do sistema privado quanto do SUS, constavam na REMUME. Porém, verificou-se que, em alguns casos, os medicamentos que não estavam elencados na REMUME foram os mais prescritos em seus respectivos grupos farmacológicos, como no caso de medicamentos para o sistema nervoso, algumas classes utilizadas no sistema cardiovascular e para o aparelho respiratório.

A Tabela 2 mostra a prevalência de prescrição de medicamentos não constantes na REMUME e os classifica conforme a existência ou não de alternativas terapêuticas disponíveis nesta lista, da mesma classe farmacológica (nível 3 ATC) ou do mesmo grupo químico (nível 4 ATC). Além dos grupos farmacológicos já identificados na Tabela 1, também foi possível demonstrar mais alguns grupos de medicamentos não constantes na REMUME e sem alternativa terapêutica disponível: diuréticos de baixo limiar com exceção das tiazidas, adrenérgicos de ação central, preparados antigotosos e colírios antiglaucomatosos.

As Tabelas 3 e 4 listam os medicamentos que foram prescritos por profissionais do SUS e do sistema privado, respectivamente, e a forma de obtenção destes medicamentos. Percebe-se que a maioria dos idosos que foi atendida pelo SUS obtém seus medicamentos no sistema público de saúde $(74,4 \%)$. E, entre os que foram atendidos no sistema privado, 14,8\% também adquiriram seus medicamentos no SUS.

A maioria dos medicamentos prescritos por profissionais do SUS consta na REMUME $(78,7 \%)$, o que demonstra que os prescritores do SUS seguem a lista dos medicamentos disponíveis no município. Já dentre as prescrições no sistema privado, apenas $47,2 \%$ são medicamentos elencados na REMUME. A Tabela 5 mostra a proporção de prescrições de medicamentos não constantes na REMUME segundo o prescritor (SUS ou do sistema privado), deixando clara a maior proporção de prescrição de medicamentos não constantes na REMUME por profissional do sistema privado.

\section{Discussão}

Os medicamentos mais utilizados foram os indicados para o sistema cardiovascular, sendo a classe farmacológica dos redutores de colesterol e triglicerídeos os mais prevalentes, apesar de não haver nenhum exemplar desta classe de medicamentos relacionado na REMUME. Todavia, apesar de algumas faltas, a lista de medicamentos selecionados está em conformidade com a maioria dos medicamentos mais prescritos no Município de Florianópolis. A principal diferença entre as prescrições é que as oriundas do SUS estão em maior conformidade com a REMUME.

O tamanho da amostra e a metodologia da pesquisa garantem que os resultados sejam representativos de todas as prescrições para idosos residentes na zona urbana do Município de Florianópolis. A automedicação não está contemplada nas análises por não ser objeto deste estudo.

Para minimizar um possível viés de memória, foram adotados procedimentos padronizados para a coleta dos dados, que incluíram a solicitação de apresentação da receita, embalagem ou bula do medicamento referido. Além disso, foram analisados somente os medicamentos prescritos, fato que minimiza o esquecimento por serem medicamentos incorporados na rotina diária do idoso.

Quase metade (46,8\%) dos idosos residentes na área urbana do Município de Florianópolis utilizou pelo menos algum medicamento para o sistema cardiovascular. Esse resultado condiz com os obtidos em outros estudos brasileiros que utilizaram a mesma metodologia do presente trabalho 12,13,14,15, com resultados variando entre $29 \%$ e $54 \%$.

As outras classes de medicamentos mais utilizadas foram, em ordem decrescente, as usadas para o trato alimentar e metabolismo (como por exemplo, os hipoglicemiantes), medicamentos para o sistema nervoso (como antidepressivos e ansiolíticos) e para doenças vasculares (antitrombóticos, como por exemplo, o ácido acetilsalicílico), o que já foi confirmado por outros estudos 12,13,14. Algumas pesquisas, porém, apontaram os medicamentos para o sistema nervoso em segundo lugar 15 .

Esses dados são explicados pela alta prevalência de doenças cardiovasculares e diabetes entre 
Descrição dos medicamentos prescritos, utilizados nos 30 dias anteriores à entrevista, por ordem decrescente do número de utilizações, conforme classificação anatômica (nível 1) e grupo farmacológico (nível 3) da Anatomical Therapeutical Chemical Index (ATC), não presentes na Relação Municipal de Medicamentos (REMUME), segundo existência (sim) ou não existência (não) de alternativa terapêutica na REMUME. Projeto EpiFloripa Idoso, Florianópolis, Santa Catarina, Brasil, 2010.

\begin{tabular}{|c|c|c|c|c|c|c|}
\hline \multirow[t]{2}{*}{ Medicamento } & \multicolumn{2}{|c|}{ Sim } & \multicolumn{2}{|c|}{ Não } & \multicolumn{2}{|c|}{ Total } \\
\hline & $\mathbf{n}$ & $\%$ & $\mathbf{n}$ & $\%$ & $\mathbf{n}$ & $\%$ \\
\hline C - Sistema Cardiovascular & 406 & 40,4 & 600 & 59,6 & 1.006 & 46,6 \\
\hline C10A - Redutores do colesterol e triglicerídeos & - & - & 432 & 100,0 & 432 & 20,0 \\
\hline C07A - Betabloqueadores & 72 & 100,0 & - & - & 72 & 3,3 \\
\hline C09C - Antagonistas da angiotensina II & 63 & 100,0 & - & - & 63 & 2,9 \\
\hline C09D - Antagonistas da angiotensina II em associações & 53 & 100,0 & - & - & 53 & 2,5 \\
\hline C01D - Vasodilatadores utilizados em doenças cardíacas & 48 & 100,0 & - & - & 48 & 2,2 \\
\hline C03B - Diuréticos de "teto baixo", excluindo as tiazidas & - & - & 45 & 100,0 & 45 & 2,1 \\
\hline C08C - Bloqueadores seletivos dos canais de cálcio com efeitos vasculares & 37 & 100,0 & - & - & 37 & 1,7 \\
\hline C09A - Inibidor da enzima conversora da angiotensina & 36 & 100,0 & - & - & 36 & 1,7 \\
\hline C08D - Bloqueadores seletivos dos canais de cálcio com efeitos cardíacos & 35 & 100,0 & - & - & 35 & 1,6 \\
\hline C02A - Antiadrenérgicos de ação central & - & - & 33 & 100,0 & 33 & 1,5 \\
\hline C03A - Diuréticos tiazídicos & 29 & 100,0 & - & - & 29 & 1,3 \\
\hline Outros & 33 & 26,8 & 90 & 73,2 & 123 & 5,7 \\
\hline A - Trato Alimentar e Metabolismo & 135 & 81,3 & 31 & 18,7 & 166 & 7,7 \\
\hline A10B - Fármacos hipoglicemiantes orais & 70 & 100,0 & - & - & 70 & 3,2 \\
\hline A02B - Medicamentos para tratamento de úlcera péptica e refluxo gastroesofageal & 39 & 100,0 & - & - & 39 & 1,8 \\
\hline Outros & 26 & 45,6 & 31 & 54,4 & 57 & 2,6 \\
\hline $\mathrm{N}$ - Sistema Nervoso & 381 & 83,6 & 75 & 16,4 & 456 & 21,1 \\
\hline N06A - Antidepressivos & 126 & 100,0 & - & - & 126 & 5,8 \\
\hline N03A - Antiepiléticos & 84 & 100,0 & - & - & 84 & 3,9 \\
\hline N05B - Ansiolíticos & 80 & 100,0 & - & - & 80 & 3,7 \\
\hline N07C - Preparações antivertiginosas & 38 & 100,0 & - & - & 38 & 1,8 \\
\hline N05A - Antipsicóticos & 32 & 100,0 & - & - & 32 & 1,5 \\
\hline Outros & 21 & 21,9 & 75 & 78,1 & 96 & 4,4 \\
\hline B - Sangue e Órgãos Formadores de Sangue & 110 & 99,1 & 1 & 0,9 & 111 & 5,1 \\
\hline B01A - Antitrombótico & 110 & 100,0 & - & - & 110 & 5,1 \\
\hline Outros & - & - & 1 & 100,0 & 1 & 0,0 \\
\hline M - Sistema Musculoesquelético & 74 & 40,2 & 110 & 59,8 & 184 & 8,5 \\
\hline M01A - Anti-inflamatórios e antirreumáticos não esteroidais & 74 & 100,0 & - & - & 74 & 3,4 \\
\hline M05B - Medicamentos que afetam a estrutura óssea e mineralização & - & - & 60 & 100,0 & 60 & 2,8 \\
\hline M04A - Preparados antigotosos & - & - & 31 & 100,0 & 31 & 1,4 \\
\hline Outros & - & - & 19 & 100,0 & 19 & 0,9 \\
\hline R - Aparelho Respiratório & 83 & 83,0 & 17 & 17,0 & 100 & 4,6 \\
\hline R03A - Adrenérgicos para inalação & 56 & 100,0 & - & - & 56 & 2,6 \\
\hline Outros & 27 & 61,4 & 17 & 38,6 & 44 & 2,0 \\
\hline S - Órgãos dos Sentidos & - & - & 52 & 100,0 & 52 & 2,4 \\
\hline S01E - Preparados antiglaucomatosos e mióticos & - & - & 42 & 100,0 & 42 & 1,9 \\
\hline Outros & - & - & 10 & 100,0 & 10 & 0,5 \\
\hline Outros $(D, H, G, J, L, P)$ & 20 & 23,3 & 66 & 76,7 & 86 & 4,0 \\
\hline Total & 1.209 & 55,9 & 952 & 44,1 & 2.161 & 100,0 \\
\hline
\end{tabular}


Descrição dos medicamentos prescritos por profissional do SUS, utilizados nos 30 dias anteriores à entrevista, por ordem decrescente do número de utilizações, conforme classificação anatômica (nível 1) e grupo farmacológico (nível 3) da Anatomical Therapeutical Chemical Index (ATC), segundo forma de obtenção. Projeto EpiFloripa Idoso, Florianópolis, Santa Catarina, Brasil, 2010.

\begin{tabular}{|c|c|c|c|c|c|c|}
\hline \multirow[t]{2}{*}{ Medicamento } & \multicolumn{2}{|c|}{ SUS } & \multicolumn{2}{|c|}{ Compra } & \multicolumn{2}{|c|}{ Total } \\
\hline & $\mathbf{n}$ & $\%$ & $\mathbf{n}$ & $\%$ & $\mathbf{n}$ & $\%$ \\
\hline C - Sistema Cardiovascular & 875 & 77,2 & 258 & 22,8 & 1.133 & 49,6 \\
\hline C09A - Inibidor da enzima conversora da angiotensina & 229 & 90,9 & 23 & 9,1 & 252 & 11,0 \\
\hline C03A - Diuréticos tiazídicos & 168 & 91,8 & 15 & 8,2 & 183 & 8,0 \\
\hline C10A - Redutores do colesterol e triglicerídeos & 50 & 35,0 & 93 & 65,0 & 143 & 6,3 \\
\hline C07A - Betabloqueadores & 111 & 87,4 & 16 & 12,6 & 127 & 5,6 \\
\hline C09C - Antagonistas da angiotensina II & 88 & 86,3 & 14 & 13,7 & 102 & 4,5 \\
\hline C08C - Bloqueadores seletivos dos canais de cálcio com efeitos vasculares & 53 & 76,8 & 16 & 23,2 & 69 & 3,0 \\
\hline C01D - Vasodilatadores utilizados em doenças cardíacas & 36 & 66,7 & 18 & 33,3 & 54 & 2,4 \\
\hline C03C - Diuréticos de alça & 50 & 94,3 & 3 & 5,7 & 53 & 2,3 \\
\hline C03D - Agentes poupadores de potássio & 27 & 81,8 & 6 & 18,2 & 33 & 1,4 \\
\hline C08D - Bloqueadores seletivos dos canais de cálcio com efeitos cardíacos & 7 & 53,8 & 6 & 46,2 & 13 & 0,6 \\
\hline C09D - Antagonistas da angiotensina II em associações & 1 & 25,0 & 3 & 75,0 & 4 & 0,2 \\
\hline Outros & 55 & 55,0 & 45 & 45,0 & 100 & 4,4 \\
\hline A - Trato Alimentar e Metabolismo & 330 & 87,8 & 46 & 12,2 & 376 & 16,5 \\
\hline A10B - Fármacos hipoglicemiantes orais & 168 & 93,3 & 12 & 6,7 & 180 & 7,9 \\
\hline A02B - Medicamentos para tratamento de úlcera péptica e refluxo gastroesofageal & 130 & 90,9 & 13 & 9,1 & 143 & 6,3 \\
\hline Outros & 32 & 60,4 & 21 & 39,6 & 53 & 2,3 \\
\hline $\mathrm{N}$ - Sistema Nervoso & 167 & 60,3 & 110 & 39,7 & 277 & 12,1 \\
\hline N02B - Outros analgésicos e antipiréticos & 70 & 67,3 & 34 & 32,7 & 104 & 4,6 \\
\hline N06A - Antidepressivos & 46 & 78,0 & 13 & 22,0 & 59 & 2,6 \\
\hline N05B - Ansiolíticos & 15 & 50,0 & 15 & 50,0 & 30 & 1,3 \\
\hline N03A - Antiepiléticos & 11 & 40,7 & 16 & 59,3 & 27 & 1,2 \\
\hline N07C - Preparações antivertiginosas & 9 & 40,9 & 13 & 59,1 & 22 & 1,0 \\
\hline Outros & 16 & 45,7 & 19 & 54,3 & 35 & 1,5 \\
\hline B - Sangue e Órgãos Formadores de Sangue & 139 & 76,8 & 42 & 23,2 & 181 & 7,9 \\
\hline B01A - Antitrombótico & 138 & 76,7 & 42 & 23,3 & 180 & 7,9 \\
\hline Outros & 1 & 100,0 & - & - & 1 & 0,0 \\
\hline M - Sistema Musculoesquelético & 50 & 54,9 & 41 & 45,1 & 91 & 4,0 \\
\hline M01A - Anti-inflamatórios e antirreumáticos não esteroidais & 44 & 63,8 & 25 & 36,2 & 69 & 3,0 \\
\hline M05B - Medicamentos que afetam a estrutura óssea e mineralização & 4 & 36,4 & 7 & 63,6 & 11 & 0,5 \\
\hline Outros & 2 & 18,2 & 9 & 81,8 & 11 & 0,5 \\
\hline H - Preparados Hormonais Sistêmicos, exceto Hormônios Sexuais & 70 & 87,5 & 10 & 12,5 & 80 & 3,5 \\
\hline H03A - Hormônios tireoidianos & 58 & 90,6 & 6 & 9,4 & 64 & 2,8 \\
\hline Outros & 12 & 75,0 & 4 & 25,0 & 16 & 0,7 \\
\hline R - Aparelho Respiratório & 38 & 47,5 & 42 & 52,5 & 80 & 3,5 \\
\hline R03A - Adrenérgicos para inalação & 16 & 47,1 & 18 & 52,9 & 34 & 1,5 \\
\hline Outros & 22 & 47,8 & 24 & 52,2 & 46 & 2,0 \\
\hline Outros $(D, G, J, L, P, S)$ & 29 & 44,6 & 36 & 55,4 & 65 & 2,8 \\
\hline Total & 1.698 & 74,4 & 585 & 25,6 & 2.283 & 100,0 \\
\hline
\end{tabular}


Descrição dos medicamentos prescritos por profissional do sistema privado, utilizados nos 30 dias anteriores à entrevista, por ordem decrescente do número de utilizações, conforme classificação anatômica (nível 1) e grupo farmacológico (nível 3) da Anatomical Therapeutical Chemical Index (ATC), segundo forma de obtenção. Projeto EpiFloripa Idoso, Florianópolis, Santa Catarina, Brasil, 2010.

\begin{tabular}{|c|c|c|c|c|c|c|}
\hline \multirow[t]{2}{*}{ Medicamento } & \multicolumn{2}{|c|}{ SUS } & \multicolumn{2}{|c|}{ Compra } & \multicolumn{2}{|c|}{ Total } \\
\hline & $\mathbf{n}$ & $\%$ & n & $\%$ & $\mathbf{n}$ & $\%$ \\
\hline C - Sistema Cardiovascular & 229 & 16,4 & 1.171 & 83,6 & 1.400 & 44,7 \\
\hline C10A - Redutores do colesterol e triglicerídeos & 17 & 6,0 & 267 & 94,0 & 284 & 9,1 \\
\hline C07A - Betabloqueadores & 40 & 22,2 & 140 & 77,8 & 180 & 5,7 \\
\hline C09A - Inibidor da enzima conversora da angiotensina & 47 & 32,6 & 97 & 67,4 & 144 & 4,6 \\
\hline C09C - Antagonistas da angiotensina II & 32 & 22,9 & 108 & 77,1 & 140 & 4,5 \\
\hline C08C - Bloqueadores seletivos dos canais de cálcio com efeitos vasculares & 15 & 16,3 & 77 & 83,7 & 92 & 2,9 \\
\hline C09D - Antagonistas da angiotensina II em associações & 2 & 2,2 & 88 & 97,8 & 90 & 2,9 \\
\hline C03A - Diuréticos tiazídicos & 36 & 40,4 & 53 & 59,6 & 89 & 2,8 \\
\hline C01D - Vasodilatadores utilizados em doenças cardíacas & 8 & 14,0 & 49 & 86,0 & 57 & 1,8 \\
\hline C03C - Diuréticos de alça & 11 & 25,0 & 33 & 75,0 & 44 & 1,4 \\
\hline C08D - Bloqueadores seletivos dos canais de cálcio com efeitos cardíacos & 2 & 4,9 & 39 & 95,1 & 41 & 1,3 \\
\hline C03D - Agentes poupadores de potássio & 5 & 23,8 & 16 & 76,2 & 21 & 0,7 \\
\hline Outros & 14 & 6,4 & 204 & 93,6 & 218 & 7,0 \\
\hline A - Trato Alimentar e Metabolismo & 105 & 24,0 & 333 & 76,0 & 438 & 14,0 \\
\hline A10B - Fármacos hipoglicemiantes orais & 47 & 22,5 & 162 & 77,5 & 209 & 6,7 \\
\hline A02B - Medicamentos para tratamento de úlcera péptica e refluxo gastroesofageal & 47 & 28,1 & 120 & 71,9 & 167 & 5,3 \\
\hline Outros & 11 & 17,7 & 51 & 82,3 & 62 & 2,0 \\
\hline N - Sistema Nervoso & 57 & 11,4 & 445 & 88,6 & 502 & 16,0 \\
\hline N06A - Antidepressivos & 28 & 17,0 & 137 & 83,0 & 165 & 5,3 \\
\hline N03A - Antiepiléticos & 9 & 10,0 & 81 & 90,0 & 90 & 2,9 \\
\hline N05B - Ansiolíticos & 2 & 2,9 & 67 & 97,1 & 69 & 2,2 \\
\hline N02B - Outros analgésicos e antipiréticos & 9 & 18,8 & 39 & 81,3 & 48 & 1,5 \\
\hline N07C - Preparações antivertiginosas & 2 & 6,7 & 28 & 93,3 & 30 & 1,0 \\
\hline Outros & 7 & 7,0 & 93 & 93,0 & 100 & 3,2 \\
\hline B - Sangue e Órgãos Formadores de Sangue & 28 & 11,6 & 213 & 88,4 & 241 & 7,7 \\
\hline B01A - Antitrombótico & 28 & 11,6 & 213 & 88,4 & 241 & 7,7 \\
\hline Outros & - & - & & - & - & - \\
\hline M - Sistema Musculoesquelético & 5 & 2,7 & 179 & 97,3 & 184 & 5,9 \\
\hline M01A - Anti-inflamatórios e antirreumáticos não esteroidais & 4 & 4,2 & 92 & 95,8 & 96 & 3,1 \\
\hline M05B - Medicamentos que afetam a estrutura óssea e mineralização & 0 & 0,0 & 49 & 100,0 & 49 & 1,6 \\
\hline Outros & 1 & 2,6 & 38 & 97,4 & 39 & 1,2 \\
\hline H - Preparados Hormonais Sistêmicos, exceto Hormônios Sexuais & 26 & 14,9 & 148 & 85,1 & 174 & 5,6 \\
\hline H03A - Hormônios tireoidianos & 26 & 16,0 & 136 & 84,0 & 162 & 5,2 \\
\hline Outros & 0 & 0,0 & 12 & 100,0 & 12 & 0,4 \\
\hline R - Aparelho Respiratório & 8 & 9,4 & 77 & 90,6 & 85 & 2,7 \\
\hline R03A - Adrenérgicos para inalação & 4 & 8,5 & 43 & 91,5 & 47 & 1,5 \\
\hline Outros & 4 & 10,5 & 34 & 89,5 & 38 & 1,2 \\
\hline Outros (D, G, J, L, P, S) & 7 & 6,4 & 103 & 93,6 & 110 & 3,5 \\
\hline Total & 465 & 14,8 & 2.669 & 85,2 & 3.134 & 100,0 \\
\hline
\end{tabular}

a população idosa 16,17 , assim como quadros de insônia, ansiedade e estados confusionais, resultando na prescrição de fármacos para o sistema nervoso 10 . Verifica-se, portanto, a importância e a necessidade de atenção das políticas públi- cas para o fornecimento de medicamentos adequados e efetivos para o tratamento das doenças cardiovasculares e para o tratamento da diabetes, assim como maior atenção na prescrição de medicamentos para o sistema nervoso central, já 
Descrição dos medicamentos utilizados nos 30 dias anteriores à entrevista, não presentes na Relação Municipal de Medicamentos (REMUME), conforme classificação anatômica (nível 1) e prescrição por profissional do SUS ou do sistema privado. Projeto EpiFloripa Idoso, Florianópolis, Santa Catarina, Brasil, 2010.

\begin{tabular}{|c|c|c|c|c|}
\hline \multirow[t]{2}{*}{ Medicamento } & \multicolumn{2}{|c|}{ SUS } & \multicolumn{2}{|c|}{ Privado } \\
\hline & $\mathrm{n}$ & $\%$ & $\mathrm{n}$ & $\%$ \\
\hline A - Trato Alimentar e Metabolismo & 25 & 15,1 & 141 & 84,9 \\
\hline B - Sangue e Órgãos Formadores de Sangue & 28 & 25,2 & 83 & 74,8 \\
\hline C - Sistema Cardiovascular & 239 & 23,8 & 767 & 76,2 \\
\hline D - Dermatológicos & 7 & 43,8 & 9 & 56,3 \\
\hline G - Aparelho Genitourinário e Hormônios Sexuais & 6 & 24,0 & 19 & 76,0 \\
\hline H - Preparados Hormonais Sistêmicos, exceto Hormônios Sexuais & 3 & 37,5 & 5 & 62,5 \\
\hline J - Anti-infecciosos Gerais para Uso Sistêmico & 1 & 14,3 & 6 & 85,7 \\
\hline L - Agentes Antineoplásicos e Imunomouladores & 8 & 33,3 & 16 & 66,7 \\
\hline M - Sistema Musculoesquelético & 40 & 21,7 & 144 & 78,3 \\
\hline $\mathrm{N}$ - Sistema nervoso & 82 & 18,0 & 374 & 82,0 \\
\hline P - Antiparasitários & 1 & 25,0 & 3 & 75,0 \\
\hline R - Aparelho Respiratório & 33 & 33,0 & 67 & 67,0 \\
\hline S - Órgãos dos Sentidos & 14 & 26,9 & 38 & 73,1 \\
\hline Total & 487 & 22,6 & 1.672 & 77,4 \\
\hline
\end{tabular}

que são os que mais causam efeitos adversos e uso inadequado nos idosos 12,18.

Dentre todos os medicamentos, o mais prescrito foi da classe farmacológica dos redutores de colesterol e triglicerídeos. Por ocasião da pesquisa esses medicamentos constavam somente no antigo Programa de Medicamentos Excepcionais 19 (atualmente denominado Componente Especializado de Assistência Farmacêutica), não relacionados na REMUME. Esses medicamentos responderam por quase $20 \%$ de todas as prescrições não constantes na REMUME, sendo o de maior peso.

Nenhum dos medicamentos que afetam a estrutura óssea e mineralização estava relacionado na REMUME no momento da coleta de dados, e somente eram disponibilizados pelo Programa de Medicamentos Excepcionais, já citado anteriormente. Tampouco outros grupos farmacológicos, como os adrenérgicos de ação central, preparados antigotosos e colírios antiglaucomatosos estavam disponíveis ou possuíam alternativa terapêutica. A prevalência do glaucoma aumenta com a idade, dobrando a cada década de vida 20 , demonstrando a importância epidemiológica da inclusão desta classe de medicamentos na lista.

Pelo conceito da Organização Mundial da Saúde (OMS) 21 (p. 14), medicamentos essenciais "são aqueles que servem para satisfazer às necessidades de atenção à saúde da maioria da população", e os medicamentos anteriormente citados se enquadram perfeitamente neste conceito. Os resultados deste estudo populacional demonstram que a demanda desses medicamentos é alta, o que sugere que eles devam ser disponibilizados na REMUME.

Vale ressaltar outro grupo de medicamentos que não aparece nas tabelas por ser pouco prevalente, mas também não possui exemplares disponíveis na REMUME nem alternativa terapêutica disponível e que deveria ser revisto para uma possível seleção: os medicamentos para o sistema genitourinário (grupo G), para tratamento da hiperplasia prostática benigna.

Destaca-se que, durante a realização da coleta de dados deste estudo, foram aprovadas novas Portarias 22,23,24 que determinam que todos os municípios do Estado de Santa Catarina forneçam o medicamento sinvastatina (redutor de colesterol e triglicerídeos), além do medicamento alendronato de sódio, pertencente à classe dos que afetam a estrutura óssea e mineralização, e o maleato de timolol, para tratamento do glaucoma. As Portarias são datadas de novembro de 2009 e o prazo para entrarem em vigor era de três meses após as suas publicações (março de 2010), porém o município somente começou o fornecimento desses medicamentos após o término do levantamento de dados deste trabalho.

Constatou-se que, dentre todos os medicamentos prescritos, a maior proporção deles é padronizada pela REMUME. Isso demonstra que 
a lista de medicamentos selecionados pelo sistema público supre as necessidades da maioria dos medicamentos que estão sendo prescritos aos idosos do município. A importância desse dado está em demonstrar que, como não é possível ao sistema de saúde fornecer todos os medicamentos disponíveis no mercado, o município teve êxito ao selecionar aqueles com eficácia comprovada, que servem de referência dentro de uma determinada classe de medicamentos. Além disso, demonstra que os profissionais prescritores, em sua maioria, conhecem e seguem a lista de medicamentos disponibilizados pelo SUS.

Porém, dentre os medicamentos utilizados para o sistema nervoso, algumas classes de antihipertensivos (como os antagonistas da angiotensina II em associações) e dos medicamentos para o sistema respiratório (como os adrenérgicos para inalação), os mais prevalentes foram os não constantes na REMUME. Isso sugere que a REMUME não está suprindo as indicações dos prescritores e/ou as necessidades dos pacientes idosos nesses casos.

Os medicamentos para o sistema nervoso são utilizados em casos em que a variabilidade da doença e do paciente influem muito na eficácia do medicamento, fazendo com que sejam prescritos tratamentos individualizados para cada caso $25,26,27$, que dificilmente podem seguir uma lista restritiva de medicamentos. Nesses casos, é importante haver várias alternativas terapêuticas disponíveis para a realização do tratamento, ou seja, não é suficiente a seleção de poucos exemplares de cada grupo farmacológico, já que não serão todas as pessoas que poderão utilizar os mesmos medicamentos. Portanto, a lista deve ser revista nesse aspecto para aumentar o número de medicamentos elencados para essas doenças.

Além disso, e ainda incluindo os antagonistas da angiotensina II em associações, uma explicação que pode ter gerado o resultado citado anteriormente é que essas classes de medicamentos foram mais prescritas por profissionais do sistema privado, que não necessariamente seguem as listas do SUS quando prescrevem os medicamentos.

Os adrenérgicos para inalação elencados na REMUME estão sendo pouco prescritos tanto no sistema privado quanto no SUS, existindo a preferência por outros representantes deste grupo químico no momento da prescrição médica. Verifica-se que constam na lista somente medicamentos utilizados para melhora dos sintomas agudos da broncoconstrição, como o salbutamol e o fenoterol, que são agonistas beta2 de ação rápida. Porém, agonistas beta2 de ação longa, como o formoterol e o salmeterol, não estão disponíveis na REMUME. Esses medicamentos, associados aos corticoesteróides inalatórios, são necessários para manutenção do tratamento dos pacientes com broncoconstrição causada por asma, doença pulmonar obstrutiva crônica (DPOC) etc. 28. Portanto, a inclusão dessa classe de medicamentos também deve ser revista. Vale informar que a associação dos medicamentos formoterol+budesonida é disponibilizada somente pelo já referido Programa de Medicamentos Excepcionais 19.

Outras causas podem estar relacionadas com a prescrição de medicamentos não constantes na REMUME. Cabe ressaltar que a maioria das pessoas entrevistadas $(87,5 \%)$ utilizava dois ou mais medicamentos concomitantemente, o que pode gerar prescrições fora da lista de medicamentos, pela necessidade de se utilizar outro tipo de medicamento para não causar interações medicamentosas.

Os resultados também permitiram demonstrar a diferença entre as prescrições no sistema privado e público, no que diz respeito à variabilidade de classes de medicamentos. Os profissionais do sistema público prescrevem mais medicamentos constantes na REMUME, o que não acontece com os prescritores do sistema privado. Isso ocorre principalmente no caso de antidepressivos, hipoglicemiantes orais, ansiolíticos, medicamentos para tratamento de úlcera péptica e refluxo gastroesofageal e betabloqueadores. Além disso, no sistema privado prescrevem-se mais os diuréticos de baixo limiar excluindo as tiazidas, e antidislipidêmicos em associações. Os motivos para essas diferenças podem ser de natureza cultural (influência da indústria farmacêutica, preferência pessoal), desconhecimento da lista do SUS e da possibilidade de acesso universal a esses medicamentos, e questões relacionadas à adesão e aos efeitos colaterais do tratamento.

Os idosos que realizaram consulta médica pelo SUS obtiveram a maioria dos seus medicamentos também no SUS. Esse resultado foi igualmente verificado em estudo no Município de Pelotas, Rio Grande do Sul, em população coberta pelo Programa Saúde da Família (PSF) em que, do total de medicamentos usados para tratar doenças crônicas, $63 \%$ foram obtidos gratuitamente e, considerando-se somente os medicamentos usados para tratar hipertensão e diabetes, o fornecimento gratuito cobriu cerca de $80 \%$ dos medicamentos usados 29. Um outro estudo, que avaliou o acesso de idosos a medicamentos para hipertensão e diabetes no Nordeste e Sul do país, mostrou que, de todos os medicamentos obtidos, $35,1 \%$ foram pagos pelos idosos e o restante $(64,9 \%)$ foi fornecido gratuitamente nas unidades de saúde ou por outros meios ${ }^{30}$. 


\section{Conclusão}

Em geral, a seleção dos medicamentos fornecidos pela REMUME Florianópolis está em conformidade com as necessidades da maioria da população de idosos do município, pois grande parte dos medicamentos mais utilizados e prescritos consta na lista.

No entanto, foram verificadas algumas falhas na seleção dos medicamentos, como a falta de algum representante das classes dos redutores de colesterol e triglicerídeos, dos que afetam a estrutura óssea e mineralização e dos antiglaucomatosos. Cabe ressaltar que, pela inclusão de exemplares dessas classes nas novas Portarias vigentes após a realização deste estudo, demonstrou-se que os resultados encontrados realmente representavam uma falha na seleção de medicamentos no SUS nesses casos.

Sabe-se que a demanda de medicamentos prescritos não é a melhor forma de avaliação de uma lista de medicamentos. Sugerimos maiores estudos, baseados em literatura científica, para a verificação da lista de medicamentos fornecidos, principalmente nos casos dos antidepressivos, antiepiléticos, ansiolíticos e adrenérgicos para inalação, que não estão sendo seguidos pelos prescritores em geral.

Este estudo deixou clara a diferença de prescrição entre os profissionais do sistema público e do privado, principalmente na prescrição de medicamentos fora das listas do SUS. Isso pode acarretar vários problemas no sistema público, sendo o exemplo mais atual e preocupante o aumento do número de ações judiciais para obtenção de medicamentos não fornecidos pelo SUS. Levando-se em consideração o conceito de medicamentos essenciais e a formulação de listas de medicamentos, não é possível ao sistema público de saúde dar conta de fornecer todos os medicamentos prescritos no sistema privado.

Demonstra-se a importância da educação médica para o conhecimento da lista dos medicamentos que são fornecidos pelo SUS, a possibilidade de acesso universal a eles e a necessidade de prescrição racional. Verifica-se que os profissionais, principalmente os que atuam em consultórios particulares, são constantemente influenciados pela indústria farmacêutica e acabam por prescrever medicamentos que poderiam ser substituídos por outros igualmente efetivos, porém de menor custo.

Também deve-se investir no esclarecimento da população sobre os medicamentos que são disponibilizados pelo SUS, e deixar claro o sentido conceitual da lista e os benefícios advindos de sua adoção. A formulação de listas de medicamentos essenciais não é aprovada por todos os setores da sociedade. A indústria farmacêutica, organizações vinculadas a agravos específicos e alguns profissionais as consideram muito restritivas ou insuficientes. Por essa razão, é importante a conscientização de que se trata de uma importante estratégia na promoção do uso racional de medicamentos, na melhoria da qualidade de atenção à saúde e da gestão dos medicamentos. Porém, ressalta-se que essa seleção deve ser baseada em evidência científica de eficácia e segurança para tornar-se confiável, além de ser periodicamente avaliada e atualizada.

\section{Resumo}

O objetivo deste trabalho foi descrever os medicamentos prescritos aos idosos residentes na zona urbana do Município de Florianópolis, Santa Catarina, Brasil, nos 30 dias anteriores à entrevista e, a partir disto, comparar com a Relação Municipal de Medicamentos (REMUME). Foi realizado um estudo transversal populacional de base domiciliar, no qual os 1.705 idosos entrevistados relataram ter utilizado o total de 5.458 medicamentos prescritos e dentro dos critérios da pesquisa, que corresponderam a 374 princípios ativos diferentes. Os medicamentos mais utilizados foram os indicados para o sistema cardiovascular, sendo a classe farmacológica dos redutores de colesterol e triglicerídeos os mais prevalentes, apesar de não haver nenhum exemplar desta classe de medicamentos selecionado na REMUME. Embora haja algumas faltas, a REMUME coincide com a maior proporção dos medicamentos prescritos no Município de Florianópolis. A principal diferença entre as prescrições é que as oriundas do SUS estão em maior conformidade com a REMUME.

Uso de Medicamentos; Idoso; Acesso aos Serviços de Saúde 


\section{Colaboradores}

M. M. Aziz contribuiu para a concepção, planejamento, análise, interpretação dos dados, elaboração do rascunho, revisão crítica do conteúdo, e aprovação da versão final do trabalho. M. C. M. Calvo contribuiu para a concepção, análise e interpretação dos dados, elaboração do rascunho e aprovação final do trabalho. E. d'Orsi contribuiu para a concepção, planejamento, análise e interpretação dos dados, elaboração do rascunho e aprovação final do trabalho.

\section{Referências}

1. Loyola Filho AI, Uchoa E, Firmo JOA, Lima-Costa MF. Influência da renda na associação entre disfunção cognitiva e polifarmácia: Projeto Bambuí. Rev Saúde Pública 2008; 42:89-99.

2. World Health Organization. The world medicines situation. Geneva: World Health Organization; 2004.

3. Fardelone CL, Branchi BA. Mudanças recentes no mercado farmacêutico. Revista da FAE 2006; 9:139-52.

4. IMS Institute for Healthcare Informatics. The global use of medicines: outlook through 2015. http:// www.imshealth.com/deployedfiles/imshealth/ Global/Content/IMS\%20Institute/Static\%20File/ Global_Use_of_Medicines_Report.pdf (acessado em 05/Jul/2011).

5. Marin N, Luiza VL, Osório-de-Castro CGS, Machado-dos-Santos S. Assistência farmacêutica para gerentes municipais. Rio de Janeiro: Organização Pan-Americana da Saúde/Organização Mundial da Saúde; 2003.

6. Wannmacher L. Medicamentos essenciais: vantagens de trabalhar com este contexto. Uso Racional de Medicamentos: Temas Selecionados 2006; 3:1-6.

\section{Agradecimentos}

Agradecemos aos técnicos do Instituto Brasileiro de Geografia e Estatística (IBGE) e da Secretaria Municipal de Saúde de Florianópolis por nos ter auxiliado a operacionalizar este estudo.
7. Departamento de Assistência Farmacêutica e Insumos Estratégicos, Secretaria de Ciência, Tecnologias e Insumos Estratégicos, Ministério da Saúde. Relação Nacional de Medicamentos Essenciais: RENAME. 7ạ Ed. Brasília: Ministério da Saúde; 2010.

8. Naves JOS, Silver LD. Evaluation of pharmaceutical assistance in public primary care in Brasília, Brazil. Rev Saúde Pública 2005; 39:223-30.

9. Instituto Brasileiro de Geografia e Estatística. Estimativas populacionais para o TCU. Estimativas da população para 1o de julho de 2009. http://www. ibge.gov.br/home/estatistica/populacao/estima tiva2009/POP2009_DOU.pdf (acessado em 03/ Dez/2009).

10. Diretoria de Atenção Primária à Saúde, Secretaria Municipal de Saúde. Protocolo de atenção à saúde do idoso. Florianópolis: Prefeitura Municipal de Florianópolis; 2011.

11. Instituto Brasileiro de Geografia e Estatística. Censo 2000. http://www.ibge.gov.br/home/estatisti ca/populacao/censo2000/default.shtm (acessado em $01 / J u l / 2008)$. 
12. Coelho Filho JM, Marcopito LF, Castelo A. Perfil de utilização de medicamentos por idosos em área urbana do nordeste do Brasil. Rev Saúde Pública 2004; 38:557-64.

13. Flores LM, Mengue SS. Uso de medicamentos por idosos em região do sul do Brasil. Rev Saúde Pública 2005 ; 39:924-9.

14. Loyola Filho AI, Uchoa E, Lima-Costa MF. Estudo epidemiológico de base populacional sobre uso de medicamentos entre idosos na região metropolitana de Belo Horizonte, Minas Gerais, Brasil. Cad Saúde Pública 2006; 22:2657-67.

15. Loyola Filho AI, Uchôa E, Firmo JOA, Lima-Costa MFF. Estudo de base populacional sobre o consumo de medicamentos entre idosos: Projeto Bambuí. Cad Saúde Pública 2005; 21:545-53.

16. Lima-Costa MF, Barreto SM, Giatti L. Condições de saúde, capacidade funcional, uso de serviços de saúde e gastos com medicamentos da população idosa brasileira: um estudo descritivo baseado na Pesquisa Nacional por Amostra de Domicílios. Cad Saúde Pública 2003; 19:735-43.

17. Benedetti TB, Petroski EL, Gonçalves LT. Condições de saúde nos idosos de Florianópolis. ACM Arq Catarin Med 2006; 35:44-51.

18. Rozenfeld S. Prevalência, fatores associados e mal uso de medicamentos entre os idosos: uma revisão. Cad Saúde Pública 2003; 19:717-24.

19. Ministério da Saúde. Portaria GM nº 2.577 de 27 de outubro de 2006. Aprova o Componente de Medicamentos de Dispensação Excepcional. Diário Oficial da união2006; 30 out.

20. Sociedade Brasileira de Glaucoma. 3o consenso brasileiro de glaucoma primário de ângulo aberto. São Paulo: Editora BestPoint/Sociedade Brasileira de Glaucoma; 2009.

21. World Health Organization. Report on the 12th expert committee on the selection and use of essential medicines. Geneva: World Health Organization; 2002. (Technical Report Series, 914).
22. Ministério da Saúde. Portaria $\mathrm{n}^{\circ} .2 .981$ de 26 de novembro de 2009. Aprova o Componente Especializado da Assistência Farmacêutica. Diário Oficial da União 2009; 30 nov.

23. Ministério da Saúde. Portaria no .2 .982 de 26 de novembro de 2009. Aprova as normas de execução e de financiamento da Assistência Farmacêutica na Atenção Básica. Diário Oficial da União 2009; 1 dez.

24. Secretaria de Estado da Saúde de Santa Catarina/ Comissão Intergestores Bipartite. Deliberação 164/CIB/10. Florianópolis: Comissão Intergestores Bipartite; 2010.

25. Souza FGM. Tratamento da depressão. Rev Bras Psiquiatr 1999; 21:18-23.

26. Ministério da Saúde. Portaria SAS/MS nº. 492, de 23 de setembro de 2010. Diário Oficial da União 2010; 24 set.

27. Associação Brasileira de Psiquiatria. Projeto diretrizes - transtornos de ansiedade: diagnóstico e tratamento. Rio de Janeiro: Associação Brasileira de Psiquiatria; 2008.

28. Ministério da Saúde. Portaria SAS/MS nº. 1.012 de 23 de dezembro de 2002. Diário Oficial da União 2002; 24 dez.

29. Bertoldi AD. Epidemiologia do acesso aos medicamentos e sua utilização em uma população assistida pelo Programa Saúde da Família [Tese de Doutorado]. Pelotas: Universidade Federal de Pelotas; 2006.

30. Paniz VMV, Fassa AG, Facchini LA, Piccini RX, Tomasi E, Thumé E, et al. Acesso gratuito a medicamentos para hipertensão e diabetes em idosos: uma realidade a ser construída. Cad Saúde Pública 2010; 26:1163-74.

Recebido em 21/Abr/2011

Versão final reapresentada em 18/Jul/2011 Aprovado em 22/Ago/2011 